\title{
Cryo-balloon catheter localization in X-Ray fluoroscopy using U-net
}

\author{
Ina Vernikouskaya ${ }^{1}\left[\right.$ Dagmar Bertsche $^{1} \cdot$ Tillman Dahme $^{1} \cdot$ Volker Rasche $^{1}$
}

Received: 11 January 2021 / Accepted: 1 April 2021 / Published online: 20 April 2021

(c) The Author(s) 2021

\begin{abstract}
Purpose Automatic identification of interventional devices in X-ray (XR) fluoroscopy offers the potential of improved navigation during transcatheter endovascular procedures. This paper presents a prototype implementation of fully automatic 3D reconstruction of a cryo-balloon catheter during pulmonary vein isolation (PVI) procedures by deep learning approaches. Methods We employ convolutional neural networks (CNN) to automatically identify the cryo-balloon XR marker and catheter shaft in 2D fluoroscopy during PVI. Training data are generated exploiting established semiautomatic techniques, including template-matching and analytical graph building. A first network of U-net architecture uses a single grayscale XR image as input and yields the mask of the XR marker. A second network of the similar architecture is trained using the mask of the XR marker as additional input to the grayscale XR image for the segmentation of the cryo-balloon catheter shaft mask. The structures automatically identified in two $2 \mathrm{D}$ images with different angulations are then used to reconstruct the cryo-balloon in 3D.

Results Automatic identification of the XR marker was successful in $78 \%$ of test cases and in $100 \%$ for the catheter shaft. Training of the model for prediction of the XR marker mask was successful with 3426 training samples. Incorporation of the XR marker mask as additional input for the model predicting the catheter shaft allowed to achieve good training result with only 805 training samples. The average prediction time per frame was $14.47 \mathrm{~ms}$ for the XR marker and $78.22 \mathrm{~ms}$ for the catheter shaft. Localization accuracy for the XR marker yielded on average 1.52 pixels or $0.56 \mathrm{~mm}$.

Conclusions In this paper, we report a novel method for automatic detection and 3D reconstruction of the cryo-balloon catheter shaft and marker from 2D fluoroscopic images. Initial evaluation yields promising results thus indicating the high potential of CNNs as alternatives to the current state-of-the-art solutions.
\end{abstract}

Keywords Cryo-balloon $\cdot$ Automatic segmentation $\cdot$ Reconstruction $\cdot$ Semi-automatic annotation $\cdot$ Unet

\section{Introduction}

Atrial fibrillation is a common heart arrhythmia associated with an increased risk of stroke. The current state-of-theart treatment option is minimally invasive catheter ablation. Cryo-balloon catheters isolate a pulmonary vein by freezing

Ina Vernikouskaya

ina.vernikouskaya@uni-ulm.de

Dagmar Bertsche

dagmar.bertsche@uni-ulm.de

Tillman Dahme

tillman.dahme@uniklinik-ulm.de

Volker Rasche

volker.rasche@uni-ulm.de

1 Department of Internal Medicine II, Ulm University Medical Center, Albert-Einstein-Allee 23, 89081 Ulm, Germany the tissue annularly around its ostium using liquid nitrogen injected into the balloon device. The planning of cryo-balloon ablations of atrial fibrillation is a crucial task for a physician as determining the correct size of the balloon catheter is required for successful isolation of each pulmonary vein $(\mathrm{PV})$. The radio-opaque marker of the cryo-balloon localized in the biplane X-ray (XR) fluoroscopy facilitates the verification of the balloon catheter position with respect to the preoperative data set of the left atrium [1]. Further intra-procedural support can be provided by visualizing the position and dimensions of the cryo-balloon catheter overlaid onto the fluoroscopic images using overlay guidance systems (OGS) [2,3]. Based on the knowledge of its 3D XR marker position, vector of the catheter shaft, and diameter, several methods for the reconstruction of a 3D model of the cryo-balloon have been introduced with reported submillimeter levels of accuracy for sphere reconstruction $[1,4,5]$. 
Also, methods to reconstruct ellipsoids requiring three views [6] or spheroids from even a single image plane [7] have been reported. The previously addressed 3D reconstruction methods will significantly benefit from automatic detection of the radio-opaque marker and the catheter shaft in the fluoroscopic images, as it has been shown for automatic $3 \mathrm{D}$ catheter tracking inside the patient's vascular tree [8] or in electrophysiology (EP) procedures $[9,10]$. Therefore, the purpose of this work is to fully automatically identify the cryo-balloon marker and the catheter shaft in 2D XR fluoroscopy to be further used for initialization of the 3D reconstruction method of the cryo-balloon, exemplarily addressed in this paper.

Although convolutional neural networks (CNN) are extensively used for catheter detection and tracking, a large amount of annotated training data is required for all deep learning approaches for pixel-level segmentation. Manual pixel-level labeling is laborious and time-consuming; thus, methods reducing the required manual interaction will greatly facilitate the development of CNN models for semantic segmentation [11].

In this work, we propose to use networks of similar encoder-decoder U-net architecture to detect separately the $\mathrm{XR}$ marker and the cryo-balloon catheter shaft in the XR images from cryo-balloon pulmonary vein isolation (PVI) procedures. For training of the networks, semiautomatically annotated training data were used. The annotated training data for marker prediction were used additionally to the catheter shaft training data for further improvement of prediction capability and simultaneous reduction of the required amount of training data.

\section{Methods}

\section{CNN model}

The proposed method utilizes an adapted CNN model of the well-known U-net architecture to segment the radioopaque marker (U-net ${ }^{\text {marker }}$ ) and the cryo-balloon catheter shaft (U-net ${ }^{\text {shaft }}$ ) separately in the XR images from PVI procedures. The adapted U-net model consists of a convolutional part downsampled with the maxpool layer and strided transposed convolutional upsampling part in combination with dropout regularization and residual connection to the output and was previously described by our group [12]. Both models were implemented in Keras and trained on a GeForce GTX 10606 GB GPU for 50 epochs with a batch size of 8 (U-net $^{\text {marker }}$ ) or 4 (U-net ${ }^{\text {shaft }}$ ) samples per pass with the adaptive moment estimation algorithm. U-net ${ }^{\text {marker }}$ uses a lossless grayscale XR image as input and predicts the radio-opaque marker as a mask (mask ${ }^{\text {marker }}$ ). U-net ${ }^{\text {shaft }}$ consists of two inputs: current lossless grayscale XR image and mask ${ }^{\text {marker }}$, and predicts the catheter shaft mask (mask ${ }^{\text {shaft }}$ ). The implemented algorithm showing the annotation and training steps with corresponding inputs/outputs, as well as predicted outputs of both models is sketched in Fig. 1.

All available XR data were pre-processed and annotated semiautomatically as described below in Annotation.

\section{Data}

Eighty-nine fluoroscopic runs from 15 different PVI patients acquired on biplane C-arm X-ray system (Allura Xper, Philips Healthcare, Best, The Netherlands) were used for development and evaluation of the proposed method. Two patients' data were acquired in monoplane mode (44 runs in total), 13 patients' data-in biplane mode (45 runs in total, on average 2 frontal and 2 lateral runs per patient). A total of 3426 XR images of $512 \times 512$ pixels resolution (all 76 available runs from 11 patients) were successfully annotated and used for training/validation of U-net ${ }^{\text {marker }}$. A total of 805 images ( 32 biplane runs from 10 patients and 2 monoplane runs from 1 patient) were used to train/validate the U-net ${ }^{\text {shaft }}$. A total of $508 \mathrm{XR}$ images (13 biplane runs from 4 patients) were used for testing both models.

\section{Annotation}

The radio-opaque marker mask (mask ${ }^{\text {marker}}$ ) was created by thresholding the signal intensity values of the XR image (Fig. 2a) within a $10 \times 10$ pixels region of interest (ROI) around the marker (Fig. 2b). A threshold value for marker detection was identified individually for each run based on the intensity values of the marker in the first frame. A single point within the marker was manually set in the first frame of each run and extended to the rectangular shaped template. ROI position through the run was automatically adjusted applying template-matching using the local sums to normalize the cross-correlation in MATLAB. Finally, a series of binary masks of the XR marker corresponding to each frame in the XR run was created (Fig. 2c). For each frame in the $\mathrm{XR}$ run, the created binary mask was overlaid on the XR image and reviewed visually for accuracy (Fig. 2b, closeup); the outliers were removed.

Catheter shaft binary mask (mask ${ }^{\text {shaft }}{ }^{\text {ctrl }}$ ) was created from a centerline model build similarly as described in [4, 13 ] as the analytical representation of the cryo-balloon catheter shaft part close to the XR marker. First, the following image pre-processing steps were undertaken: each 8-bit integer grayscale XR image (Fig. 3a) was inverted, histogram equalized, enhanced applying a hybrid Hessian-based filter [14], binarized, and skeletonized. The resulting image can be seen in Fig. 3b.

After pre-processing, the search area for the catheter shaft in the skeletonized image was reduced to $50 \times 50$ pixels $\mathrm{ROI}$ 


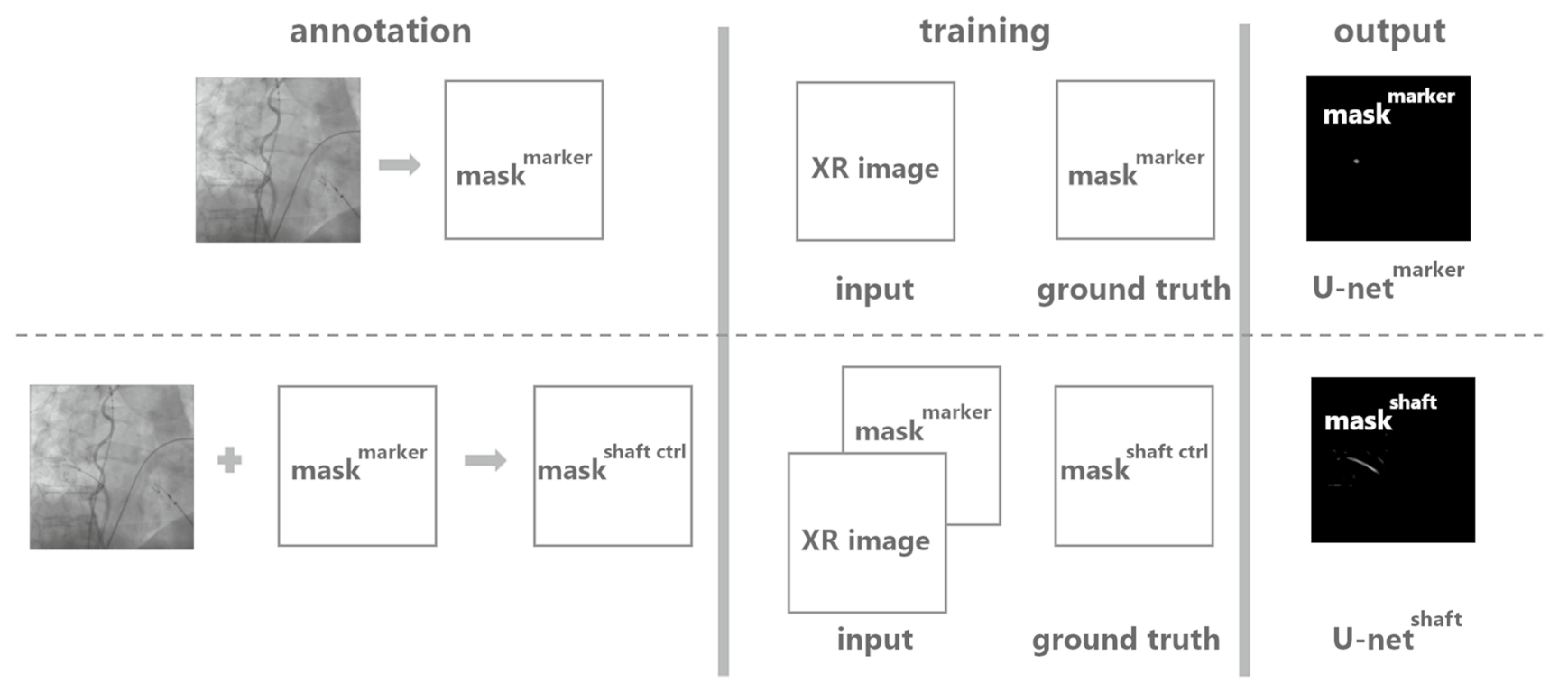

Fig. 1 Method processing algorithm subdivided into annotation, training, and output steps. Annotation step: the mask of the cryoballoon marker mask ${ }^{\text {marker }}$ is obtained from the original XR image to be processed by the U-net ${ }^{\text {marker}}$; the mask of the cryo-balloon catheter shaft centerline mask ${ }^{\text {shaft ctrl }}$ is obtained from the original XR image and its corresponding mask ${ }^{\text {marker }}$ to be processed by the U-net ${ }^{\text {shaft }}$. Training step: U-net ${ }^{\text {marker }}$ is trained on the image sample consisting of original XR image and its corresponding mask ${ }^{\text {marker }}$ as ground truth; $\mathrm{U}$-net ${ }^{\text {shaft }}$ is trained on the image sample consisting of original XR image and its corresponding mask ${ }^{\text {marker }}$ as inputs and mask ${ }^{\text {shaft ctrl }}$ as ground truth. Output step: mask ${ }^{\text {marker }}$ and mask ${ }^{\text {shaft }}$ are predicted by the U-net ${ }^{\text {marker }}$ and U-net ${ }^{\text {shaft }}$ correspondingly as images containing contours of specific pixel values against background
Fig. 2 Semiautomatic annotation of the cryo-balloon marker: a original XR image; b XR frame with a rectangular template of $10 \times 10$ pixels set around the XR marker (red box) and overlaid thresholded pixels corresponding to the segmented marker (incl. close-up of the respective region of interest); $\mathbf{c}$ resulting binary mask mask ${ }^{\text {marker }}$
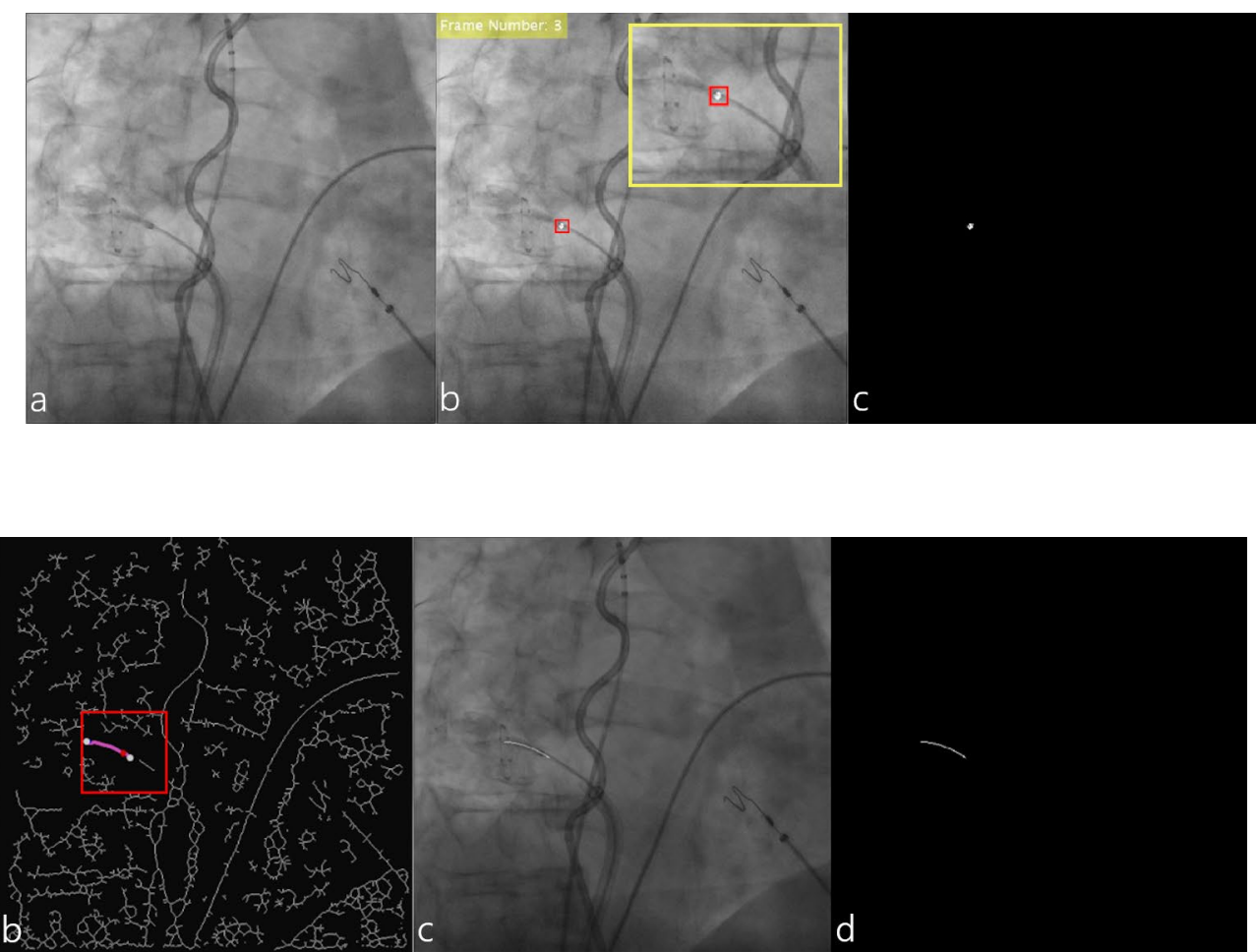

Fig. 3 Semiautomatic annotation of the cryo-balloon catheter shaft: a original XR image; b skeletonized binary image with the analytical graph (magenta) built within the $50 \times 50$ pixels ROI (red rectangular box) around the seed point (red point) and two end nodes (white points) indicating the longest path closest to the seed point and interpreted as a catheter shaft; $\mathbf{c}$ XR frame with overlaid resulting spline fitted longest path providing the centerline of the catheter shaft; $\mathbf{d}$ resulting binary mask mask ${ }^{\text {shaft ctrl }}$ 
around the marker (Fig. 3b, red rectangular box). The ROI center is given by the center of the minimum-area bounding rectangle of the contour provided by the mask ${ }^{\text {marker }}$ and will be further considered as a seed point (Fig. 3b, red point). To close small gaps, a closing operation was performed before skeletonization. The skeleton within the search area was then transformed into a single gapless graph (Fig. 3b, magenta points) initialized by the seed point. The shortest paths between all end nodes of the graph were computed by Dijkstra's algorithm. From the resulting paths, the longest path closest to the seed point located within an initially predefined catheter orientation dependent interval was considered as estimate for the catheter shaft (Fig. 3b, all nodes between two white points). The resulting centerline was then obtained by spline fitting of the computed path and overlaid onto the XR image for visual reviewing of the segmentation accuracy (Fig. 3c). In case of failure, the procedure was redone with another pre-defined interval. An exemplarily resulting binary mask mask ${ }^{\text {shaft ctrl }}$ generated for the training of U-net ${ }^{\text {shaft }}$ is shown in Fig. 3d. The complete algorithm was implemented in Python using OpenCV and SciPy libraries for image processing and NetworkX library for building the graphs.

\section{Post-processing and evaluation}

Generated outputs of both models-mask ${ }^{\text {marker }}$ and

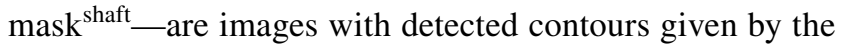

specific nonzero pixel values on the black background. To obtain a single seed point corresponding to XR marker from predicted mask ${ }^{\text {marker }}$ thresholded at $20 \%$ of maximum intensity, the centroid of the detected contour was calculated using image moments in OpenCV. For accuracy evaluation, the average Euclidean distance between the calculated seed point and centroid of the annotated marker contour was calculated. To compute the catheter shaft centerline, the mask $^{\text {shaft }}$ was thresholded at $1.5 \%$ of maximum intensity and skeletonized. Resulting centerline coordinates were obtained by spline fitting of the longest path computed for a graph built for the contour initialized by the computed seed point.

\section{$3 \mathrm{D}$ reconstruction}

The resulting seed point and spline fitted centerline for each $\mathrm{XR}$ image were used for 3D reconstruction of the cryoballoon marker and catheter shaft from two views. The reconstructed 3D marker position and catheter shaft direction vector were aligned with the previously constructed 3D model of the $28 \mathrm{~mm}$-sized cryo-balloon designed according to Arctic Front Advance Pro ${ }^{\mathrm{TM}}$ specifications (Medtronic, Minneapolis, USA), including the balloon ellipsoid, catheter shaft with tip, and radio-opaque marker (Fig. 4). With the known geometry of the XR system for each specific view, the 3D position of the radio-opaque marker given by the seed point in each view (Fig. 4, black points) was reconstructed based on epipolar geometry [15]. Created

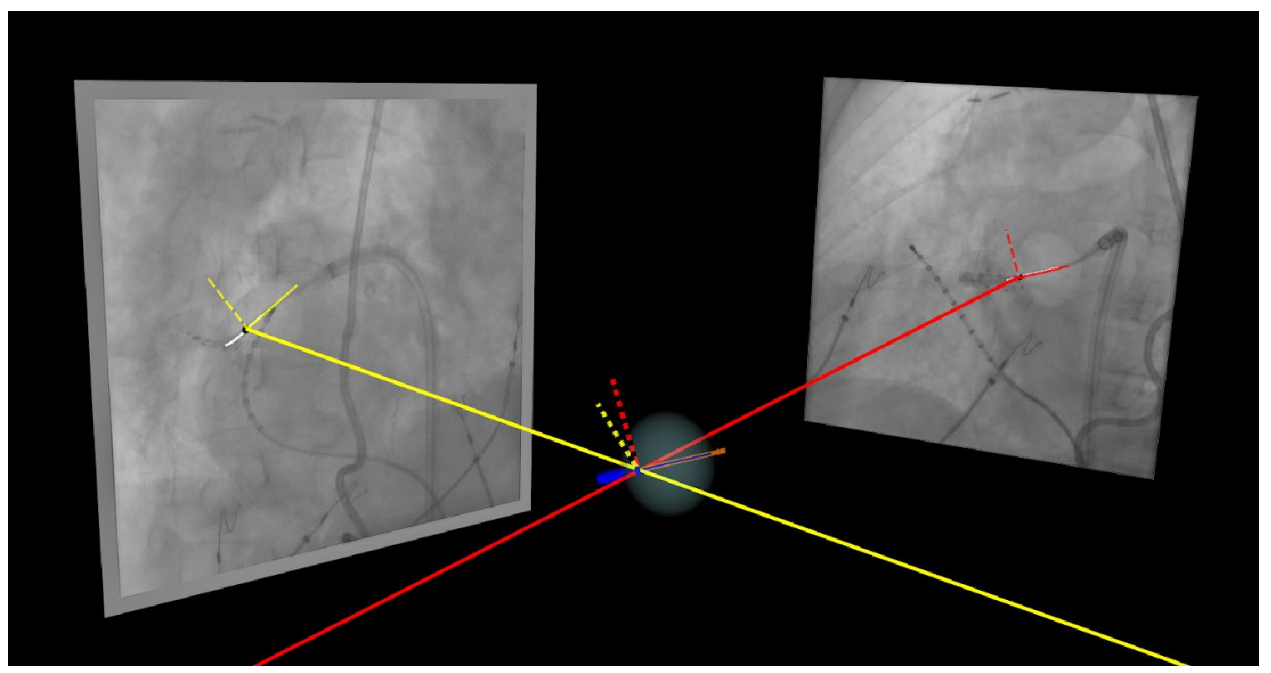

Fig. 4. 3D reconstruction of the automatically detected seed points (black points in both XR images) and catheter shaft centerlines (white lines in both XR images) from frontal view acquired in RAO30 $0^{\circ}$ orientation (red lines) and lateral view acquired in $\mathrm{LAO} 40^{\circ}$ orientation (yellow lines) and alignment with the previously constructed $28 \mathrm{~mm}$ sized cryo-balloon model. Solid red and yellow lines represent projection vectors for frontal and lateral views, respectively. Light red and yellow lines on both XR images represent respective line fitted centerlines. Dashed red and yellow lines on both XR images represent respective orthonormals, whereas dotted red and yellow lines represent transferred orthonormals initialized by the reconstructed 3D marker position aligned with the cryo-balloon model marker (dark blue small ellipsoid). Solid orange line represents resulting catheter orientation vector aligned with the cryo-balloon model shaft (dark blue line) 
centerlines in both biplane images (Fig. 4, white lines) were line fitted (Fig. 4, red light vector on frontal view and yellow light vector on lateral view) using minimal averaged Euclidean distance to obtain the associated unit direction vector. Applying the cross-product on the fitted lines and the corresponding projection vector (Fig. 4, red and yellow solid lines correspondingly), orthonormals (Fig. 4, red and yellow dashed lines) were obtained. The catheter orientation (Fig. 4, orange) resulted from the cross-product of both orthonormals, transferred according to projection geometry to the previously determined 3D marker position (Fig. 4, red and yellow dotted lines).

Since no 3D data were available as ground truth, the result of 3D reconstruction was back-projected into the image planes and the deviation of the re-projected XR marker and direction vector was calculated using the Euclidean distance between the seed points and re-projected reconstructed XR markers.

\section{Results}

The XR marker of the cryo-balloon could be visually detected in 394 ( $78 \%$ of cases) test samples, whereas 3426 image samples were used for training and validation. A total of 805 training/validation samples were required to achieve $100 \%$ visual detectability of the catheter shaft (Table 1). As expected, the accuracy of the method is limited by the presence of interfering features, e.g., injected contrast agent overlaying the target structures, other catheters, vessel-like structures, and electrodes, in the image field of view.

Even if the marker could not be detected automatically with U-net ${ }^{\text {marker }}$ (Table 1, Test runs 2, 3, 9, and 13), incorporating the annotated mask ${ }^{\text {marker }}$ as additional input for U-net ${ }^{\text {shaft }}$ yielded accurate detection of the part of the cryoballoon catheter shaft close to the seed point.

The average time to predict the whole mask of $512 \times 512$ pixels resolution was $14.47 \mathrm{~ms}$ for the XR marker and $78.22 \mathrm{~ms}$ for the catheter shaft.

Despite an average deviation of 1.52 pixels or $0.56 \mathrm{~mm}$, an accurate overlap between the centroids of the contours provided by the predicted XR marker mask and the ground truth mask could be achieved for all test data sets where the XR marker was detected (Fig. 5).

In direct comparison with the image processing algorithms utilized for the creation of the annotated training data, where the Hessian filter was often not able to manage the appearance of gaps in the near vicinity of the seed point, the detection of the catheter shaft with U-net was flawless (Fig. 6).

Figure 7 demonstrates an example of projection of the constructed cryo-balloon model aligned to the 3D structures (XR marker and direction vector) reconstructed from the automatically detected seed points and catheter centerlines in two views acquired in $\mathrm{RAO}^{\circ} 0^{\circ}$ on the frontal C-arm (Fig. 7a) and LAO40 ${ }^{\circ}$ on the lateral C-arm (Fig. 7b) onto these views. The aligned $28 \mathrm{~mm}$-sized cryo-balloon model fits the corresponding marker and catheter shaft in the XR images with the deviation of back-projected marker of

Table 1 Summary of the results from test runs provided by U-net ${ }^{\text {marker }}$ and U-net ${ }^{\text {shaft }}$

\begin{tabular}{|c|c|c|c|c|c|c|c|c|}
\hline \multirow{2}{*}{$\begin{array}{l}\text { XR test run } \\
\text { number }\end{array}$} & \multirow[t]{2}{*}{ C-arm } & \multirow{2}{*}{$\begin{array}{l}\text { Projection } \\
\text { orienta- } \\
\text { tion }\end{array}$} & \multirow{2}{*}{$\begin{array}{l}\text { Number of } \\
\text { frames in XR } \\
\text { run }\end{array}$} & \multicolumn{3}{|l|}{ U-net ${ }^{\text {marker }}$} & \multicolumn{2}{|l|}{ U-net ${ }^{\text {shaft }}$} \\
\hline & & & & $\begin{array}{l}\text { Number of } \\
\text { frames with } \\
\text { detected marker }\end{array}$ & $\begin{array}{l}\text { Prediction time } \\
\text { per frame }(\mathrm{ms})\end{array}$ & $\begin{array}{l}\text { Average Euclid- } \\
\text { ean distance } \\
(\mathrm{pxl})\end{array}$ & $\begin{array}{l}\text { Number of } \\
\text { frames with } \\
\text { detected cath- } \\
\text { eter shaft }\end{array}$ & $\begin{array}{l}\text { Prediction time } \\
\text { per frame (ms) }\end{array}$ \\
\hline 1 & frontal & $\mathrm{RAO}^{\circ} 0^{\circ}$ & 58 & 58 & 11.58 & 1.22 & 58 & 58.73 \\
\hline 2 & frontal & $\mathrm{RAO}^{\circ} 0^{\circ}$ & 49 & 0 & 12.12 & - & 49 & 63.78 \\
\hline 3 & lateral & $\mathrm{LAO} 40^{\circ}$ & 19 & 0 & 14.80 & - & 19 & 93.75 \\
\hline 4 & lateral & $\mathrm{LAO} 40^{\circ}$ & 54 & 54 & 11.86 & 1.12 & 54 & 60.76 \\
\hline 5 & frontal & $\mathrm{RAO}^{\circ} 0^{\circ}$ & 33 & 33 & 13.73 & 1.15 & 33 & 77.18 \\
\hline 6 & frontal & $\mathrm{RAO}^{\circ} 0^{\circ}$ & 19 & 19 & 15.63 & 2.77 & 19 & 94.57 \\
\hline 7 & lateral & $\mathrm{LAO}^{\prime} 0^{\circ}$ & 36 & 36 & 13.02 & 1.38 & 36 & 74.22 \\
\hline 8 & lateral & $\mathrm{LAO} 40^{\circ}$ & 10 & 10 & 28.13 & 1.5 & 10 & 148.44 \\
\hline 9 & frontal & $\mathrm{RAO}^{\circ} 0^{\circ}$ & 41 & 17 & 12.58 & 2.6 & 41 & 70.88 \\
\hline 10 & frontal & $\mathrm{RAO}^{\circ} 0^{\circ}$ & 87 & 87 & 11.85 & 1.19 & 87 & 44.72 \\
\hline 11 & lateral & $\mathrm{LAO} 40^{\circ}$ & 42 & 42 & 12.28 & 0.78 & 42 & 66.59 \\
\hline 12 & frontal & $\mathrm{RAO}^{\circ} 0^{\circ}$ & 38 & 38 & 15.63 & 1.5 & 38 & 72.37 \\
\hline 13 & lateral & $\mathrm{LAO} 40^{\circ}$ & 22 & 0 & 14.91 & - & 22 & 90.91 \\
\hline Average & & & & & 14.47 & 1.52 & & 78.22 \\
\hline
\end{tabular}




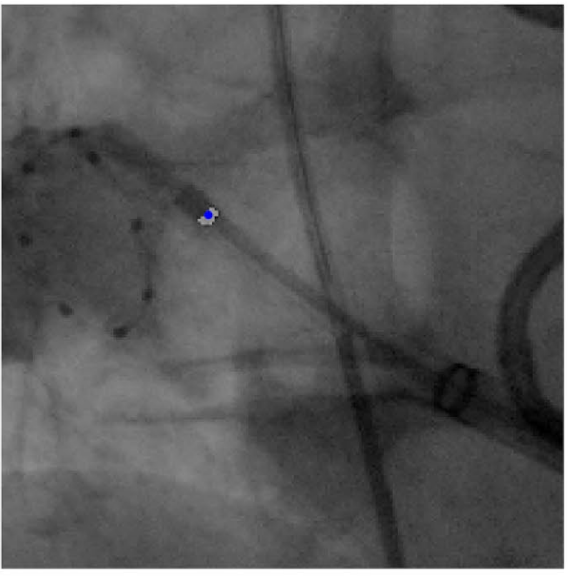

a

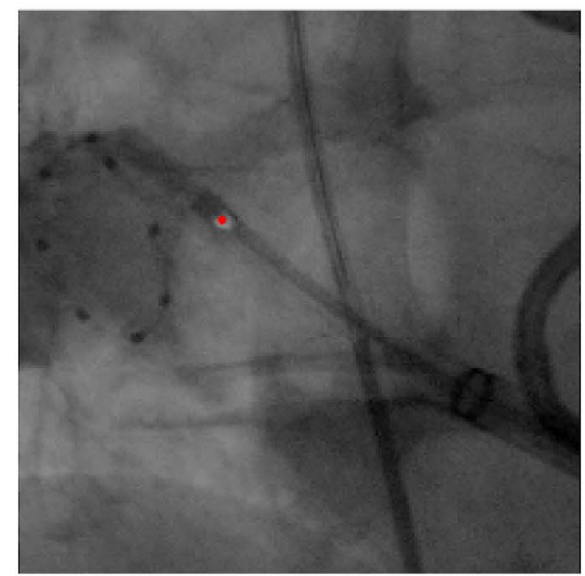

C

Fig. 5 Evaluation of the XR marker prediction accuracy from test run 1: cutout of the annotated mask ${ }^{\text {marker }}$ (a) or predicted mask ${ }^{\text {marker }}$ (c) overlaid on original XR image with the colored point indicating the centroid of the annotated marker contour as ground truth (blue)

$0.33 \mathrm{~mm}$ or 0.9 pixels in frontal and $0.28 \mathrm{~mm}$ or 0.77 pixels in lateral view in this concrete example.

\section{Discussion and conclusions}

The introduced method yields the possibility of automatic detection of the cryo-balloon catheter shaft and its marker in 2D fluoroscopic images using convolutional neural networks for localization of the cryo-balloon in 3D. The proposed annotation methods enable an automatic generation of the training data. However, for ensuring annotation fidelity, or the centroid of the predicted contour (red); plot of the predicted centroid's horizontal (b) and vertical (d) coordinates overlaid on the ground truth coordinates

manual reviewing of the accuracy is required. Especially in the case of centerline generation, the review implicates time-consuming manual effort. This effort could be, however, reduced by incorporating the mask ${ }^{\text {marker }}$ as additional input for the U-net ${ }^{\text {shaft }}$ and thus generation of less annotated data required for successful end-to-end training of U-net ${ }^{\text {shaft }}$.

The method is still facing some limitations in the detection of XR marker and catheter shaft in case of injected contrast agent overlaying the target structures and difficult catheter orientations (e.g., highly angulated catheters). This is most likely due to the very limited availability of similar training data in which proper annotation was neither 

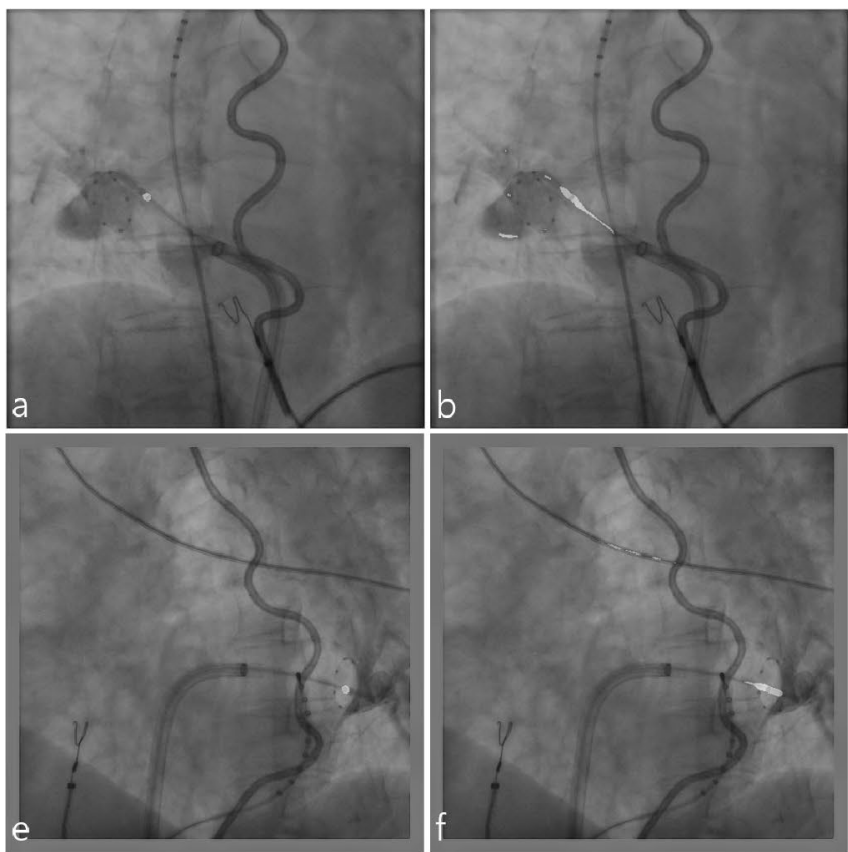

Fig. 6 Exemplary results of test run 1 (a-d) and test run 4 (e-h): a, e output of U-net ${ }^{\text {marker }}$ overlaid on original XR image; $\mathbf{b}$, $\mathbf{f}$ output of U-net ${ }^{\text {shaft }}$ overlaid on original XR image; $\mathbf{c}, \mathbf{g}$ the post-processed binary image representing the skeleton of the image outputted by

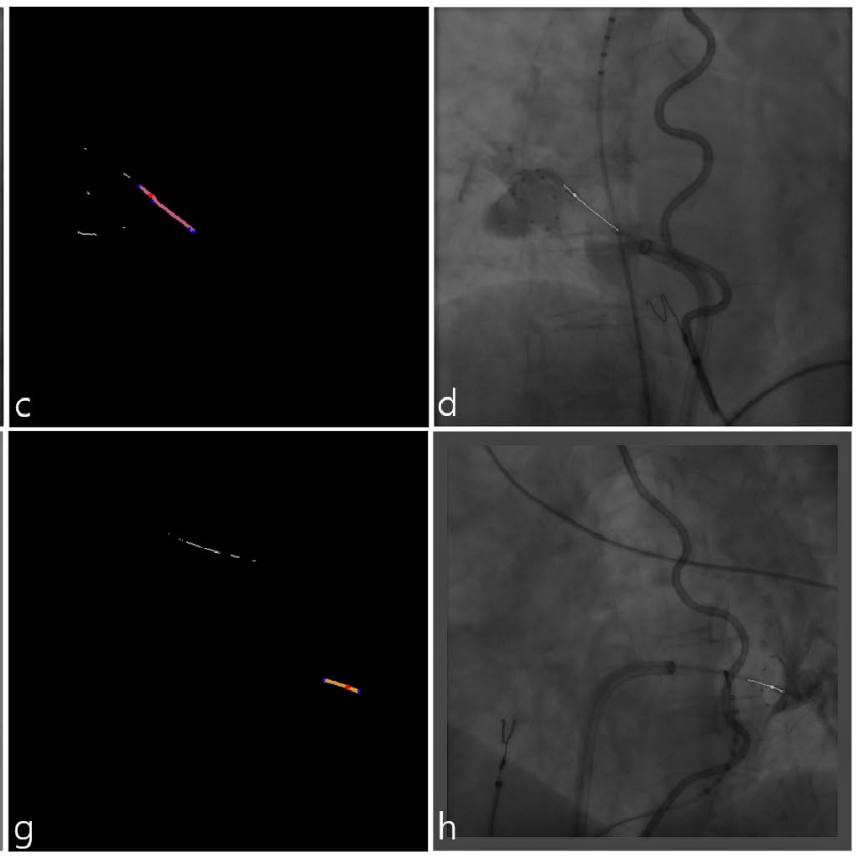

U-net ${ }^{\text {shaft }}$ with the overlaid graph and a center of the contour detected by U-net ${ }^{\text {marker }}$ (red point); $\mathbf{d}$, h resulting seed point and spline fitted centerline overlaid on original XR image

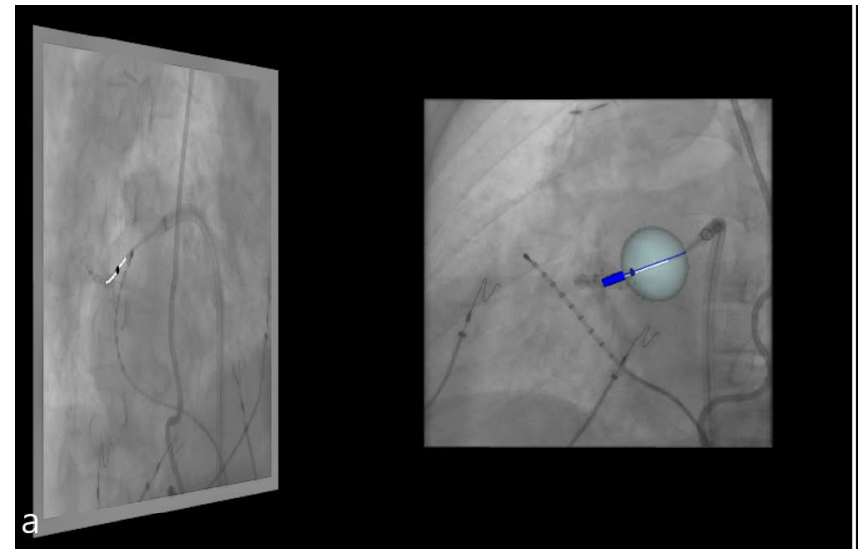

Fig. 7 Projection of the constructed $28 \mathrm{~mm}$-sized cryo-balloon model with ellipsoidal balloon (light blue) and catheter shaft (dark blue) with the tip (dark blue cylinder) and XR marker (dark blue small ellipsoid) onto $\mathrm{RAO}^{\circ} 0^{\circ}$ view acquired on the frontal $\mathrm{C}$-arm (a)

successful with the automatic approach nor manually. Moreover, the approach to uniquely identify the respective XR marker and catheter shaft in case of multiple detected regions in the predicted masks should be developed.

$3 \mathrm{D}$ reconstruction of the cryo-balloon was successful by combining the $3 \mathrm{D}$ reconstruction of the $\mathrm{XR}$ marker using epipolar geometry with the reconstruction of the 3D directional vector of the catheter shaft. For successful

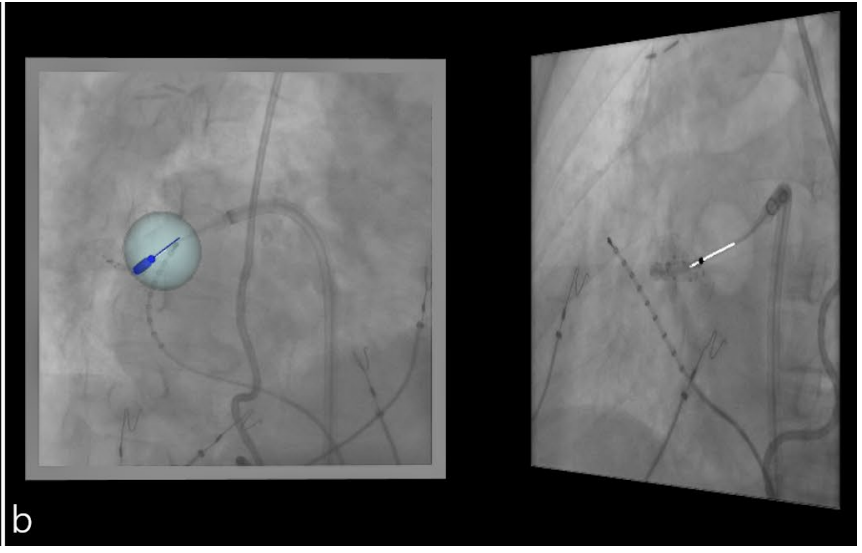

and $\mathrm{LAO} 40^{\circ}$ view acquired on the lateral C-arm (b). Automatically detected seed points are shown as black points in both XR images; catheter shaft centerlines are shown as white lines in both XR images

$3 \mathrm{D}$ reconstruction, detection of the XR marker and catheter shaft in both views should be guaranteed by the neural networks. The accuracy of the reconstruction method is limited by the calibration accuracy of the biplane XR system and is restricted to no or very low catheter shaft angulation, as only a direction vector fitted to the centerline spline is considered for reconstruction. 
Acknowledgements This work was supported by the project "Multimodal 3D Navigation System for Transvascular Interventions (TRANSNAV)" funded by German Federal Ministry of Education and Research (Contact No. BMBF-13GW0372C). Responsibility for the content of this publication lies with the authors.

Funding Open Access funding enabled and organized by Projekt DEAL.

\section{Declarations}

Conflict of interest The authors declare that they have no financial and personal relationships with other people or organizations that could inappropriately influence and/or bias this work.

Ethical Approval The imaging protocol used for human studies conforms to the ethical guidelines of the 1975 Declaration of Helsinki as reflected in a priori approval by the institution's ethics committee (Arrhythmia Register Ulm).

Informed consent Written and verbal informed consent was obtained from all individual subjects.

Open Access This article is licensed under a Creative Commons Attribution 4.0 International License, which permits use, sharing, adaptation, distribution and reproduction in any medium or format, as long as you give appropriate credit to the original author(s) and the source, provide a link to the Creative Commons licence, and indicate if changes were made. The images or other third party material in this article are included in the article's Creative Commons licence, unless indicated otherwise in a credit line to the material. If material is not included in the article's Creative Commons licence and your intended use is not permitted by statutory regulation or exceeds the permitted use, you will need to obtain permission directly from the copyright holder. To view a copy of this licence, visit http://creativecommons.org/licenses/by/4.0/.

\section{References}

1. Kurzendorfer T, Mewes PW, Maier A, Strobel N, Brost A (2016) Cryo-balloon catheter localization based on a support-vectormachine approach. IEEE Trans Med Imaging 35(8):1892-1902

2. Bourier F, Fahrig R, Wang P, Santangeli P, Kurzidim K, Strobel N, Moore T, Hinkel C, Al-Ahmad A (2014) Accuracy assessment of catheter guidance technology in electrophysiology procedures. J Cardiovasc Electrophysiol 25(1):74-83

3. Kowalewski CAB, Rodrigo M, Brodt C, Haddad F, Wang PJ, Narayan SM (2020) Novel three-dimensional imaging approach for cryoballoon navigation and confirmation of pulmonary vein occlusion. Pacing Clin Electrophysiol PACE 43(3):269-277
4. Hoffmann M, Brost A, Jakob C, Bourier F, Koch M, Kurzidim K, Hornegger J, Strobel N (2012) Semi-automatic catheter reconstruction from two views. MICCAI 2012. Lecture Notes in Computer Science. pp 584-591

5. Bourier F, Brost A, Kleinoeder A, Kurzendorfer T, Koch M, Kiraly A, Schneider HJ, Hornegger J, Strobel N, Kurzidim K (2012) Navigation for fluoroscopy-guided cryo-balloon ablation procedures of atrial fibrillation. Proc SPIE Med Imaging 2012:8316

6. Ma S, Li L (1996) Ellipsoid reconstruction from three perspective views. Proc Int Conf Pattern Recognit 1:344-348

7. Wokes DS, Palmer PL (2020) Perspective reconstruction of a spheroid from an image plane ellipse. Int $\mathrm{J}$ Comput Vis 90(3):369-379

8. Ambrosini P, Smal I, Ruijters D, Niessen WJ, Moelker A, Walsum TV (2017) A hidden markov model for 3D catheter tip tracking with 2D X-ray catheterization sequence and 3D rotational angiography. IEEE Trans Med Imaging 36(3):757-768

9. Brost A, Liao R, Strobel N, Hornegger J (2010) Respiratory motion compensation by model-based catheter tracking during EP procedures. Med Image Anal 14(5):695-706

10. Brost A, Wimmer A, Liao R, Hornegger J, Strobel N (2010) Catheter tracking: filter-based vs. learning-based. In: Goesele M, Roth S, Kuijper A, Schiele B, Schindler K (eds) Pattern recognition. DAGM 2010. Lecture Notes in Computer Science 6376

11. Gherardini M, Mazomenos E, Menciasse A, Stoyanov D (2020) Catheter segmentation in X-ray fluoroscopy using synthetic data and transfer learning with light U-nets. Comput Methods Progr Biomed 192:105420

12. Vernikouskaya I, Rottbauer W, Rasche V (2020) Real-time catheter tip segmentation and localization in 2D x-ray fluoroscopy using deep convolutional neural network. Int J Comput Assist Radiol Surg 15(Suppl 1):S67-S69

13. Ambrosini P, Ruijters D, Niessen WJ, Moelker A, van Walsum T (2017) Fully automatic and real-time catheter segmentation in X-ray fluoroscopy. MICCAI 2017. Lect Notes Comput Sci 10434:577-585

14. Ng CC, Yap MH, Costen N, Li B (2015) Automatic wrinkle detection using hybrid hessian filter. In: Cremers D, Reid I, Saito H, Yang MH (eds) Computer vision-ACCV 2014. ACCV 2014. Lecture Notes in Computer Science 9005

15. Vernikouskaya I, Bertsche D, Rottbauer W, Rasche V (2020) 3D-Xguide: open-source X-ray navigation guidance system. Int J Comput Assist Radiol Surg. https://doi.org/10.1007/ s11548-020-02274-0

Publisher's Note Springer Nature remains neutral with regard to jurisdictional claims in published maps and institutional affiliations. 